\title{
4D Scanning Electron Confocal Imaging and Visualization of Projected Bloch States
}

\section{Andrew Barnum and Mark Williamson}

Thermo Fisher Scientific, Hillsboro, Oregon, United States

For many years scanning confocal electron microscopy (SCEM) has been as a technique with the tantalizing possibility of achieving improved focal-plane localization over conventional through-focal imaging and without incurring the experimental difficulties and long acquisition times associated with tomography [1]. In this technique, the microscope is operated in an imaging STEM mode - rather than diffraction STEM - with a convergent electron probe focused on the sample and the scattered probe image focused again through the projection system onto the detector. Both probe and image Cs correctors are required to ensure that the probe is very small and that the same electrons in the probe on the sample are accurately refocused onto the detector. Previous work has relied on a dedicated pinhole aperture placed close to a diode detector (or scintillator with a photomultiplier tube) to remove out-of-focus scattering [2]. An image is recorded by scanning the beam and recording the intensity on the unscattered, in-focus portion of the electron probe. In this setup the size of the aperture ideally determines the resolution of the image in $\mathrm{z}$, parallel to the electron beam.

The recent rapid development of fast pixelated detectors, it has become possible to record the intensity of the probe directly and apply virtual apertures afterwards to generate a SCEM image. Using this technique, the authors have been able to generate ranges of bright-field, dark-field, and high angle dark field types of images using the images of the probe. In the figure, image (b) for region of the silicon finFET in (a) was generated by using a bright-field virtual aperture form the 4D-SCEM dataset. The resolution of these images can be shown to be on the order of typical STEM resolutions, with atomic resolution easily achieved.

Inspection of individual probe images reveals further detail not originally expected. As can be seen in the $4 \times 4$ probe tableau in figure (c), the probe images used to create the lattice image of silicon show peak splitting and merging based on the probe position relative to the lattice. This effect can be explained as a channeling effect of depth-dependent $1 \mathrm{~s}$ bound eigenstates [3]. The authors are not aware of previous experimental observation of this binding, and it opens the possibility of deconvolution methods tailored specifically for high-resolution SCEM. The use of 4D-SCEM to image these projection effects may be capable of providing similar crystallographic data to more traditional 4D-STEM, but without suffering from its relatively poor electron counting statistics due to the intensity inherent to the refocused beam. 

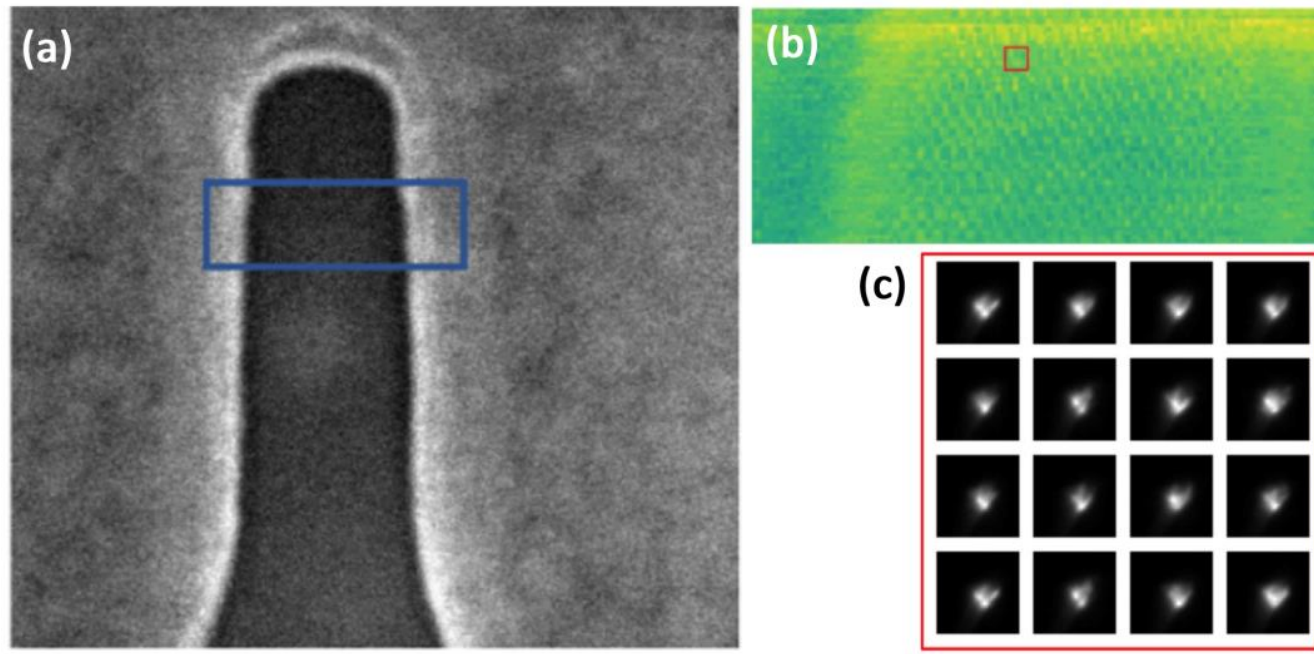

Figure 1. (a) $14 \mathrm{~nm}$ silicon finFET (orientated as $\langle 110\rangle$ ) device from commercially available processor. Region scanned using 4D-SCEM highlighted by the blue rectangle (b) Virtual BF aperture image from the 4D-SCEM data set (c) For the red box in (b), a 4x4 selection of probe image is shown, highlighting the effect of probe splitting as the probe crosses the silicon lattice.

\section{References}

[1] Nellist, P. D, G. Behan, A. I Kirkland, C. J. D Hetherington, and University of Oxford, Oxford Ox1 3ph Department of Materials. "Confocal Operation of a Transmission Electron Microscope with Two Aberration Correctors." Applied Physics Letters 89, no. 12 (2006): Applied Physics Letters, 18 September 2006, Vol.89(12).

[2] Frigo, S., Levine, Zaluzec, Materials Science Division, Northern Arizona Univ, Nist, and Argonne National Lab. "Sub-micron Imaging of Buried Integrated Circuit Structures Using Scanning Confocal Electron Microscopy." Appl. Phys. Lett 81, no. 11 (2002): 2112-2114.

[3] Hillyar, S. and Silcox, J. (1995). Detector geometry, TDS and strain in ADF STEM. Ultramicroscopy, 58, 6 